\title{
Evaluation OF STRATEgies OVER STATIC CODE ANALYSis TOOLS
}

\author{
Danilo Nikolic, Sara Havzi, Dusanka Dakic, Teodora Lolic \& Darko Stefanovic
}
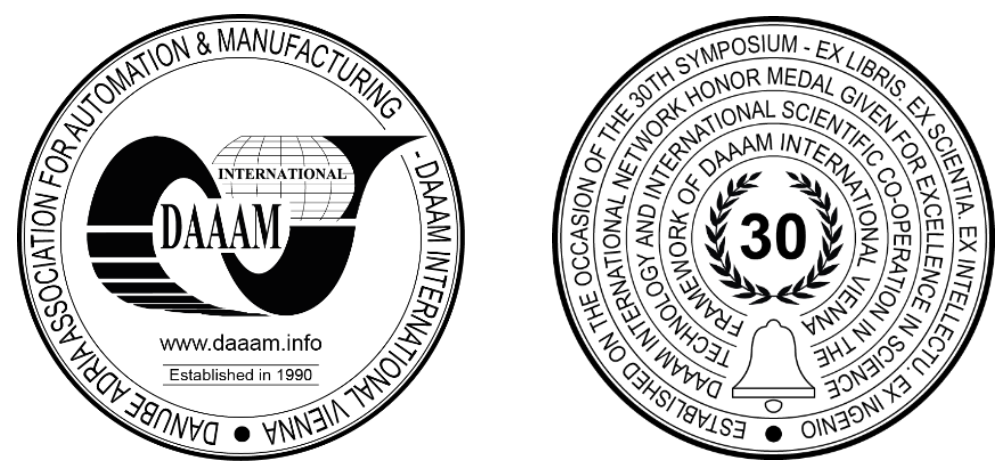

This Publication has to be referred as: Nikolic, D[anilo]; Havzi, S[ara]; Dakic, D[usanka]; Lolic, T[eodora] \& Stefanovic, D[arko] (2021). Evaluation of Strategies over Static Code Analysis Tools, Proceedings of the 32nd DAAAM International Symposium, pp.0477-0485, B. Katalinic (Ed.), Published by DAAAM International, ISBN 978-3-90273433-4, ISSN 1726-9679, Vienna, Austria

DOI: $10.2507 / 32$ nd.daaam.proceedings.069

\begin{abstract}
Source code quality is a key factor in any software product and requires constant inspection and supervision. Static code analysis tools are being increasingly used to improve code quality. Static code analysis is a valid way to infer the behavior of a program without executing it. Many tools allow static analysis in different programming languages, different frameworks, and detecting different defects in the source code. In this paper, based on the identified strategies over static code analysis tools, an experiment was conducted to evaluate the quality of the identified strategies based on established metrics. The aim of this paper is to present, analyze and evaluate the application of different strategies over different tools for static code analysis.
\end{abstract}

Keywords: static code analysis; tools; strategies; evaluation;

\section{Introduction}

Static analysis is used to maintain and improve the quality of the source code. Static analysis of program code has been used since the early 1960s to optimize the operation of compilers [1]. Later, it proved useful for debugging tools, as well as for software development frameworks. Various forms of application of information technologies are studied at the Faculty of Technical Sciences, such as [2], [3], [4], [5], [6], information technologies find application in areas such as [7], [8] too, in this paper, the application of information technologies and tools to static code analysis will be described.

Tools for static analysis have rapidly matured in the last decade, they have evolved from simple lexical analysis to employ much more complex techniques. Static analysis tools are used to produce reports and highlight specific deviations from the code quality requirements. These tools, on the other hand, do not allow for automated source code modifications. It is up to the software developers to decide whether or not to alter the composition of previously written code. A static code review tool assists software developers by creating a report that details the cause of a specific flaw as well as how to fix it. A systematic literature review in the field of static code analysis was performed as the first step in this research [9], and various static code analysis tools, programming languages for which they have support, and the types of defects they identify were presented and analyzed. The second step in the research was to identify different strategies for using static code analysis tools. Strategies are identified and described in [10]. 
The aim of this paper is to conduct an experiment over the identified strategies from [10] to analyze the use of different strategies over different tools for static code analysis and to evaluate strategies based on accuracy metrics and time required for implementation. The rest of the paper is organized as follows. Section 2 introduces fundamental principles of static code analysis tools; Section 3 describes related work based on a conducted systematic literature review; The methodology used to conduct the research is described in Section 4; Section 5 presents an experiment of the use of different strategies over static code analysis tools and Section 6 presents results and discussion of the conducted experiment and section 7 concludes the paper and suggests future research.

\section{Static Code Analysis}

This section will present how static code analysis is carried out, which tools are useful in the process, as well as what are the false positive and false negative results

\subsection{Review of the source code}

Code review is used to improve the software product maintenance process, increasing its reliability and security. Code review activity can reduce the number of required updates in source code and can be easily integrated into the software development lifecycle, so more experienced software developers often grow this activity in their work. However, manual review of source code takes a very long time [11]. In order to perform an effective manual code review, reviewers must know all possible flaws and inaccuracies before carefully checking all source code. As a substitute for manual source code review, the use of a static code analysis tool is recommended.

\subsection{Static code analysis tools}

Static code analysis is useful not just for optimizing the compiler's operation (which is what it was designed for), but also for detecting defects and potential flaws. It is possible to build software that will assist developers in understanding the behavior of a program and identifying numerous flaws in the program without having to run it [1]. Static analysis tools can often point to the root cause of a security problem, not just one of its symptoms, by examining the code itself. This is especially important if you want to make sure that any defects are fixed.

Static code analysis tools scan source code for a specific set of patterns or rules, similar to how antivirus software detects viruses. Practitioners can use more advanced tools to add new rules to a set of predefined ones. If patterns or rules do not specify such behavior, these tools will be unable to detect an error. The ability to identify common programming defects is the most valuable feature of a static code analysis tool.

Static analysis is suitable for identifying problems for several reasons [11]:

- Static analysis tools apply checks thoroughly and consistently, without any prejudice that the programmer may have about which parts of the code are "interesting" from a security perspective or which parts of the code can be easily performed by dynamic testing. It is very important that the code review is done without the subjective feeling of the programmer. Therefore, unbiased analysis can be valuable, which static code analysis tools provide.

- By examining the code itself, static analysis tools can often point to the root cause of a security problem, not just one of its symptoms. This is especially important to ensure that defects are corrected.

- Static analysis can find errors in early development, even before the program is run for the first time. Finding an error early not only reduces the cost of fixing the error, but a cycle of quick feedback can help guide the work of the programmer: The programmer has the opportunity to correct errors that he was not previously aware could even happen. Attack scenarios and code construct information used by the static analysis tool act as the means of transmitting knowledge.

- When analysis detects a new type of attack, static analysis tools make it easy to recheck a large portion of the code to detect where a new attack might occur. Some security bugs have been in the software for years before they were discovered, assessing outdated code of newly discovered types of defects is invaluable.

\subsection{False positives and False negatives}

Software developers point out that these tools produce too many bad results, these results fall into two categories [1]: 1. False Positives.

2. False Negatives.

False positive results occur when static code analysis tools report a defect in a program's source code that does not actually exist. A large number of false positive results can cause difficulties in operation. Not only is searching for a list of false positives tedious, but a software developer who needs to review a long list of false positives can easily overlook the important positive results found among them [12]. False positive results are certainly undesirable, but from a security perspective, false negative results are a much bigger problem. A false negative result implies that there is a defect in the source code of the program that the tool does not report. 
When it comes to false positive results, they only expect software developers to review the list of detected defects for a long time. Whereas, when it comes to false-positive results, they imply that the source code still contains defects, even after the static analysis procedure has been completed. This leads to a false sense of security after generating a report that there are no defects in the source code and that the quality of the code is good, even though there are false negative results [12].

Paper [13] investigated the impact of false positive alarms generated by a static code analysis tool. The researchers discovered that, while the developers were effective at recognizing true positives, their false positive detection rates were no better than chance, and their experience had no bearing on false positive classification accuracy. The same research team utilized a single static code analysis method to assess four commercial Ericsson products in a more recent study [14]. The findings revealed that the majority of the tool's output was unrelated to security and that false positives were a problem. Surprisingly, just 37.5 percent of false positives were correctly classified as such by the developers, according to the study. Furthermore, it showed that a high number of false positives harmed the development process not only because of the time spent classifying them but also because of the risk of "correcting" incorrectly classified false positives [14]. Most static code analysis tools display both false positives and false negatives. However, during the period of development of these tools, the number of errors was getting significantly smaller and these tools were able to generate more reliable results.

\section{Related Work}

As the first step in the research, a systematic literature review in the field of static analysis of code and its tools was conducted. A paper describing the process of the systematic literature review was published in [9]. The paper review was conducted using Barbara Kitchenham's guidelines for a systematic review of software engineering literature [15], with the aim of presenting the most widely used resources, through programming languages for which they have support, and defects they detect.

Based on the set inclusion criteria, by reviewing abstracts and titles, 22 primary studies were included in the literature review. Some of the representative examples of the included studies are [12], [16], [17], [18], [19]. The tools for static code analysis that are most commonly used in research on different programming languages and detecting various defects were also examined as part of this study. The majority of static code analysis tools support the most widely used programming languages in the industry. This systematic review examines the most widely used static code analysis tools in science, focusing on the types of defects they detect and the programming languages they support. Six tools most commonly used in research by the review are presented in Fig. 1.

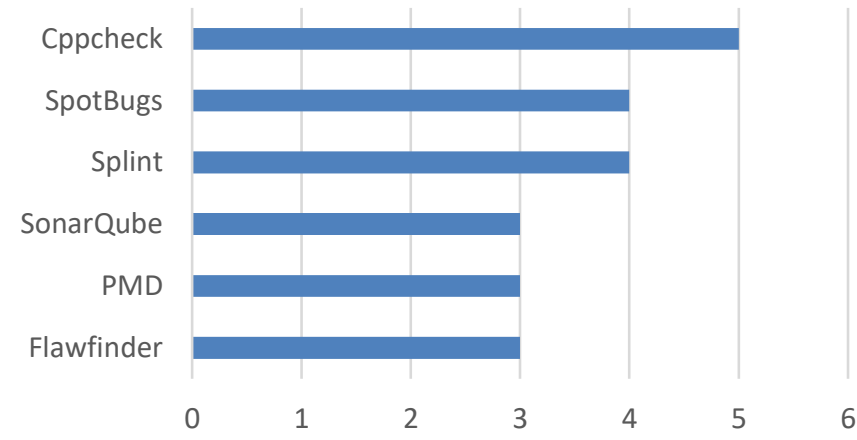

Fig. 1. The most common static code analysis tools presented in primary studies [9]

Based on the conducted literature review, the tools that will be used to conduct an experiment in this research have been selected. In addition to the literature review, support for the Java programming language and the possibility of including tools in the framework are provided as a criterion for selecting tools. Also, another research was conducted on the selected tools, in which these tools were evaluated by the DESMET method for evaluation of software tools [20]. The process of evaluation by the DESMET method and feature analysis of selected tools are described in [21].

\section{Methodology}

Based on the systematic review of the literature described in section 3 of this paper, four static code analysis tools were selected and used in the experiment.

The static code analysis tools used in the experiment are:

1. SonarQube,

2. SpotBugs,

3. PMD,

4. Checkstyle. 
Two strategies and a total of four sub-strategies for the application of static analysis tools over program source code have been identified. Those are [10]:

1. Running a static analysis tool over a completed software project $-\mathbf{S 1}$.

1.1. Starting the static analysis tool after correcting all defects $-\mathbf{S 1 . 1}$

1.2. Running the static analysis tool after correcting any defect $-\mathbf{S 1 . 2 .}$

2. Running a static analysis tool during software project development $-\mathbf{S 2}$.

2.1. Starting static analysis tools by external tool calling during software project development - S2.1.

2.2. Running static analysis tools by including tools in the development environment in which the software project is developed $-\mathbf{S 2 . 2}$.

The research [10] described in detail how the proposed strategies were identified and elaborated on the defined steps for the implementation of these strategies in the static code analysis review cycle.

The research questions, based on which strategies will be analyzed, are:

RQ1: Is there a strategy for implementing the static code analysis process that gives the best results?

RQ2: Are the identified strategies applicable to all tools used in the experiment?

RQ3: Are some strategies faster to implement than others?

RQ4: Do some strategies give more accurate results than others?

Based on the research questions, an experiment of the application of all strategies over the selected tools for static code analysis was conducted. The metrics observed during the experiment were set based on defined research questions so that the analysis of the obtained results within the metrics could lead to conclusions and answers to the research questions. The source code of the project [22] written in the Java programming language (version 1.8), within the development environment Eclipse, was used to conduct the experiment, which is a condition for the selection of tools within this experiment.

\section{Experiment}

The experiment, which was performed on selected static code analysis tools, aims to compare the results of different strategies when applied to different static code analysis tools.

To compare the application of strategies over these tools, the following metrics were used:

- Time required to eliminate all defects detected by the static analysis tool - metrics measured in minutes required to eliminate all defects in the project. This metric was set up with the aim of answering research questions RQ3 and RQ1. Unit of time used - minute.

- A total number of detected defects - a metric that implies the total number of detected defects in the source code regardless of the moment of detection of defects during the process of static code analysis. This metric is set as a reference for other metrics.

- A total number of false positive results - defects detected by tools that do not need or cannot be eliminated in the project. This metric is set with the aim of answering research questions RQ4 and RQ1.

- A total number of false negative results - defects that are of great importance for the project and were not detected by the tool. This metric was set up with the aim of answering RQ4 and RQ1 research questions.

Identified strategies and sub-strategies were applied to each tool according to the defined steps described in [10] and during the implementation of this experiment, the obtained results were recorded in relation to the set metrics for each tool and for each strategy.The results of applying strategies to SonarQube are shown in Table 1.

\begin{tabular}{|l|l|l|l|l|}
\hline Tool & \multicolumn{4}{|l|}{ SonarQube } \\
\hline Metrics/Strategies & $\boldsymbol{S 1 . 1}$ & $\boldsymbol{S 1 . 2}$ & $\boldsymbol{S 2 . 1}$ & $\boldsymbol{S 2 . 2}$ \\
\hline Time (min) & 51 & 78 & 43 & 33 \\
\hline Defects & 42 & 40 & 41 & 51 \\
\hline False positives & 2 & 0 & 3 & 10 \\
\hline False negatives & 0 & 0 & 2 & 1 \\
\hline
\end{tabular}

Table 1. Implement strategies over SonarQube 
The results of applying strategies to SpotBugs are shown in Table 2.

\begin{tabular}{|c|c|c|c|c|}
\hline Tool & \multicolumn{4}{|c|}{ SpotBugs } \\
\hline Metrics/Strategies & $\boldsymbol{S 1 . 1}$ & $\boldsymbol{S 1 . 2}$ & $\boldsymbol{S 2 . 1}$ & $\boldsymbol{S 2 . 2}$ \\
\hline Time (min) & 28 & 53 & 25 & 20 \\
\hline Defects & 22 & 19 & 24 & 30 \\
\hline False positives & 3 & 0 & 6 & 12 \\
\hline False negatives & 0 & 0 & 1 & 1 \\
\hline
\end{tabular}

Table 2. Implement strategies over SpotBugs

The results of applying strategies to PMD are shown in Table 3.

\begin{tabular}{|c|c|c|c|c|}
\hline Tool & \multicolumn{4}{|c|}{ PMD } \\
\hline Metrics/Strategies & $\mathbf{S 1 . 1}$ & $\mathbf{S 1 . 2}$ & $\boldsymbol{S 2 . 1}$ & $\boldsymbol{S 2 . 2}$ \\
\hline Time (min) & 140 & 161 & 98 & 95 \\
\hline Defects & 127 & 122 & 131 & 135 \\
\hline False positives & 14 & 10 & 20 & 26 \\
\hline False negatives & 1 & 2 & 3 & 3 \\
\hline
\end{tabular}

Table 3. Implement strategies over PMD

The results of applying strategies to Checkstyle are shown in Table 4.

\begin{tabular}{|c|c|c|c|c|}
\hline Tool & \multicolumn{4}{|c|}{ Checkstyle } \\
\hline Metrics/Strategies & $\boldsymbol{S 1 . 1}$ & $\boldsymbol{S 1 . 2}$ & $\boldsymbol{S 2 . 1}$ & $\boldsymbol{S 2 . 2}$ \\
\hline Time (min) & 122 & 128 & 96 & 88 \\
\hline Defects & 224 & 224 & 238 & 242 \\
\hline False positives & 18 & 20 & 35 & 39 \\
\hline False negatives & 0 & 2 & 3 & 3 \\
\hline
\end{tabular}

Table 4. Implement strategies over Checkstyle

In order to evaluate the results achieved by the selected tools by applying the strategies, all the tools will be presented together and the strategies identified with the modified metrics.

Now, the metrics will be labeled and interpreted as follows:

1. Average time required to eliminate one defect, expressed in seconds - ATR.

2. A total number of identified defects in the project - ND.

3. Share of false positive results in the total number of defects - FPR.

4. Share of false negative results in the total number of defects - FNR.

Aggregate results on selected tools, strategies, and defined metrics are shown in Table 5. 


\begin{tabular}{|c|c|c|c|c|c|}
\hline \multirow{2}{*}{ Tool } & \multirow{2}{*}{ Strategies } & \multicolumn{4}{|c|}{ Metrics } \\
\hline & & $A T R$ & $N D$ & $F P R$ & FNR \\
\hline \multirow{4}{*}{ SonarQube } & S1.1 & 73 & 42 & 4.8 & 0 \\
\hline & $\mathrm{S} 1.2$ & 117 & 40 & 0 & 0 \\
\hline & S2.1 & 63 & 41 & 7.3 & 4.9 \\
\hline & S2.2 & 39 & 51 & 19.6 & 2 \\
\hline \multirow{4}{*}{ SpotBugs } & S1.1 & 76 & 22 & 13.6 & 0 \\
\hline & $\mathrm{S} 1.2$ & 167 & 19 & 0 & 0 \\
\hline & S2.1 & 62 & 24 & 25 & 4.2 \\
\hline & S2.2 & 40 & 30 & 40 & 3.3 \\
\hline \multirow{4}{*}{ PMD } & S1.1 & 66 & 127 & 11 & 0.8 \\
\hline & $\mathrm{S} 1.2$ & 79 & 122 & 8.2 & 1.6 \\
\hline & S2.1 & 45 & 131 & 15.2 & 2.3 \\
\hline & S2.2 & 42 & 135 & 19.3 & 2.2 \\
\hline \multirow{4}{*}{ Checkstyle } & S1.1 & 30 & 224 & 8 & 0 \\
\hline & S1.2 & 34 & 224 & 9 & 0.9 \\
\hline & S2.1 & 24 & 238 & 14.7 & 1.3 \\
\hline & S2.2 & 21 & 242 & 16.1 & 1.2 \\
\hline
\end{tabular}

Table 5. Implement strategies over static code analysis tools

\section{Results and discussion}

An experiment within this research, which implies the application of the identified strategies, was successfully conducted on all selected tools (RQ2). In order to answer the research question RQ3, the combined results related to the metric of the average time required to eliminate one defect will be observed. The aim of this paper is not to compare tools, but to compare strategies, and the results of the ATR metrics presented in Table 5 of this research will be calculated as the average time overall the tools used for a particular strategy. The obtained result is presented in Fig. 2.

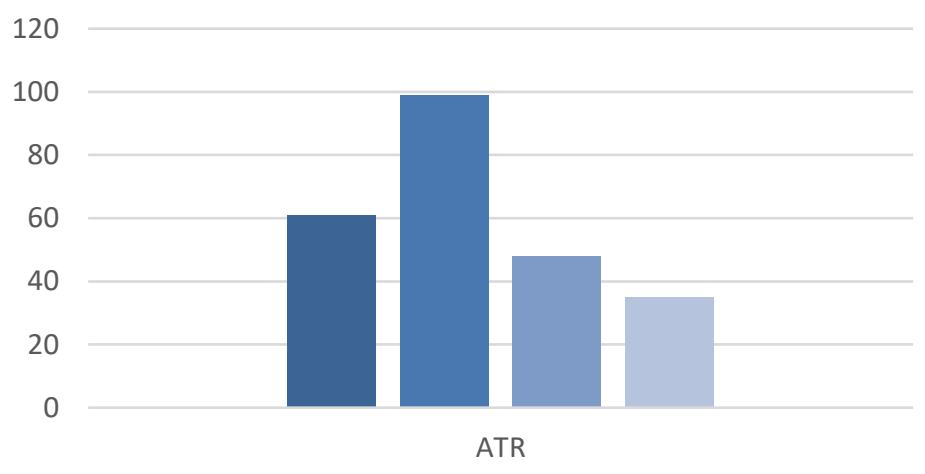

$\square \mathrm{S} 1.1 \square \mathrm{S} 1.2 \square \mathrm{S} 2.1 \square \mathrm{S} 2.2$

Fig. 2. Average time required to eliminate one defect overall tools according to the identified strategies 
Based on the presented results, it can be concluded that the S2.2 strategy is the fastest to implement. For the RQ4 the same analysis of the results should be made as for the previous question, only now the metrics of the share of false positive defects and false negative defects in the total result of the number of defects are observed. These two metrics will be combined to analyze this result because both affect the accuracy of strategy implementation. The obtained results are presented in Fig. 3.

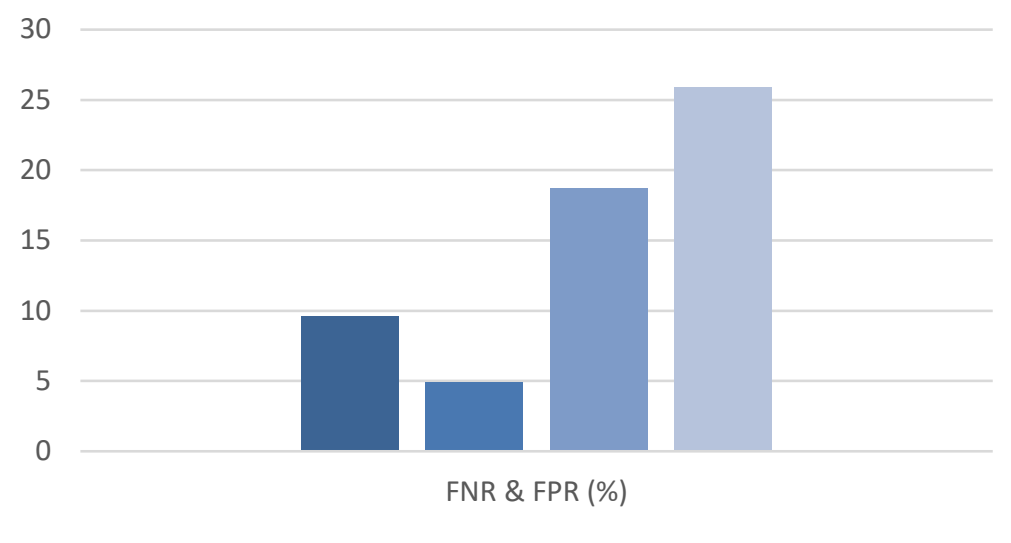

口 $1.1 \quad \mathrm{~S} 1.2 \quad \mathrm{~S} 2.1-\mathrm{S} 2.2$

Fig. 3. Percentage of false positive and false negative results in the total number of defects overall tools according to the identified strategies

Based on the presented results, it can be concluded that strategy S2.2 has the highest share of false positive and false negative results in the total number of defects detected by the tools. On the other hand, strategy S1.2 generates the smallest number of such results (RQ4). The research question RQ2 can be answered in the affirmative, that is, the identified strategies have been successfully applied to all tools. This issue also has a clearly defined limitation, as the implementation of the strategy is checked only by the tools used in this research.

In order to answer the first research question RQ1, it is necessary to define what is the best result of the implementation of the strategy. Based on the defined metrics in the paper, the best strategy is the one that gives the most accurate results, in terms of the percentage of false positives and false negatives in the total number of detected defects, and the one with the fastest implementation, that is the one with the lowest elimination time. These two metrics were set up and compared in Fig. 4.

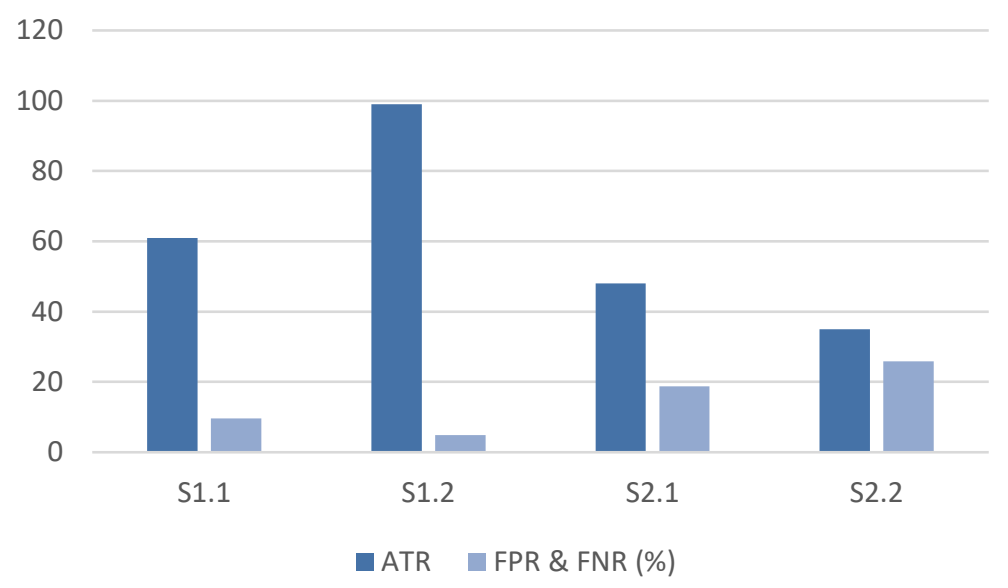

Fig. 4. Comparison of strategy implementation speed results and percentage of false positive and false negative results in the total number of identified defects

Based on the presented results, it can be concluded that strategies that are faster to implement over the tools used to generate a larger number of defects identified as FPR and FNR and strategies that are slower to implement generate a smaller number of erroneous results. The answer to research question RQ1 is that if both criteria are considered together, it is not possible to label one of the strategies as the strategy that gives the best results. It is concluded that the choice of strategy depends on the purpose of using the static code analysis tool. 


\section{Conclusion and further research}

Static code analysis tools are increasingly finding their application and are increasingly used in the daily work of software developers in various positions. Also, these tools find their application in educating future software developers about potential defects that may occur in the code, as well as their elimination by reengineering the source code.

New and improved tools are being developed every day, offering their customers an ever-improving service by building trust in the results of static analysis and enabling them to avoid certain code defects in a quality way and make their software solution better. This is especially important since the lower use of static code analysis tools is caused precisely by the user's distrust of these tools and the time required to conduct such analyses over the source code of the program. When it comes to distrust in the results of static analysis, they primarily refer to the generation of false positive and false negative results. By developing and improving these tools, the percentage of errors is reduced and these tools give better results and therefore are used more often.

Based on the conducted experiment on these strategies, certain advantages and disadvantages were noticed on the basis of defined metrics. By analyzing and comparing strategies, it is defined which strategies are faster to implement (RQ3), and which strategies give more accurate results (RQ4). In further work, it is necessary to analyze which of the identified strategies are most often used by software developers, students, and other users within different positions by conducting a questionnaire on users of static code analysis tools on the strategies they use.

The authors limit themselves to the possibility of the existence of other strategies that are not identified in this paper and are not the subject of this research. It is also necessary to conduct additional research (on other tools, different programming languages, different projects) in order to determine the existence of other strategies and sub-strategies. It is also necessary to confirm the possibility of implementing the identified strategies on other tools and software projects. In conclusion, the use of static code analysis tools and continuous work on improving and maintaining the quality of source code provides better quality software solutions and represents a step forward in software development.

\section{References}

[1] A. Moller \& I. Schwartzbach (2019). "Static program analysis"

[2] M. Arsenovic, S. Sladojevic, A. Anderla \& D. Stefanovic (2018). "Deep neural network ensemble architecture for eye movements classification," in 2018 17th International Symposium on INFOTEH-JAHORINA, INFOTEH 2018 - Proceedings, Jahorina

[3] D. Beric, S. Havzi, T. Lolic, N. Simeunovic \& D. Stefanovic (2020). "Development of the MES software and Integration with an existing ERP Software in Industrial Enterprise," in 2020 19th International Symposium INFOTEH-JAHORINA, INFOTEH 2020 - Proceedings, Jahorina

[4] D. Beric, D. Stefanovic, I. Cosic \& B. Lalic (2018). “The implementation of ERP and MES Systems as a support to industrial management systems," International Journal of Industrial Engineering and Management, vol. 9, no. 2, pp. 77-86

[5] D. Dakic, D. Stefanovic, I. Cosic, T. Lolic \& M. Medojevic (2018). “ Business Process Mining Application: A Literature Review," in Proceedings of the 29th DAAAM International Symposium, Vienna

[6] M. Jokic, D. Gracanin \& B. Lalic (2019). "Sustainable Low Cost and High-Quality Supply Chain Assurance-A Systematic Literature Review," in Proceedings of the 30th DAAAM International Symposium, Vienna, Austria

[7] M. Blahova, V. Mach, L. Pavlik, M. Hromada \& M. Ficek (2019). “ The Information Security to Software of Crisis Management," in Proceedings of the 30th DAAAM, Vienna

[8] A. Suleykin \& P. Panfilov (2019). "Implementing Big Data Processing Workflows Using Open Source Technologies," in Proceedings of the 30th DAAAM International Symposium, Vienna

[9] D. Stefanović, D. Nikolić, D. Dakić, I. Spasojević \& S. Ristić (2020). "Static code analysis tools: A systematic Literature Review," in Proceeding of of 31st DAAAM International Symposium, Vienna

[10] D. Stefanović, D. Nikolić, S. Havzi, T. Lolić \& D. Dakić (2020). "Identification of strategies over tools for static code analysis," in Proceedings of 9th International Conference On Engineering and Technology , Krabi

[11] J. West \& B. Chess (2007). "Secure programming with static code analysis," Pearson education

[12] K. Goseva-Popstojanova \& A. Perhinschi (2015). "On the capability of static code analysis to detect security vulnerabilities," Information and Software Technology, vol. 68, pp. 18-33

[13] K. P. B. C. L. L. D. Baca (2009). "Static code analysis to detect software security vulnerabilities," in International Conference on Availability, Reliability and Security

[14] B. C. K. P. L. L. D. Baca (2013). "Improving software security with static automated code analysis in an industry setting," in Software - Pract

[15] B. Kitchenham (2004). "Procedure for Undertaking Systematic Reviews," Keele University, Computer Science Department

[16] A. Kaur \& R. Nayyar (2020). "A Comparative Study of Static Code Analysis tools for Vulnerability Detection in C/C++ and JAVA Source Code," Procedia Computer Science, vol. 171, no. 2019, pp. 2023-2029

[17] D. Marcilio, C. Furia, R. Bonifacio \& P. Gustavo (2020). "SpongeBugs: Automatically generating fix suggestions in response to static code analysis warnings," The Journal of Systems \& Software, vol. 168, 
[18] P. Nunes, I. Medeiros, J. Fonseca, N. Neves, M. Correia \& M. Vieira (2019). "An empirical study on combining diverse static analysis tools for web security vulnerabilities based on development scenarios," Computing, vol. 101, no. 2, pp. 161-185

[19] N. Meghanathan (2013). "Identification and removal of software security vulnerabilities using source code analysis: a case study on a java file writer program with password validation features," Journal of Software, vol. 8, no. 10

[20] B. Kitchenham \& S. Kubjnab (2000). "DESMET: A method for evaluating Software Engineering methods and tools"

[21] D. Nikolić, D. Stefanović, D. Dakić, S. Sladojević \& S. Ristić (2021). “Analysis of the Tools for Static Code Analysis," in Proceedings of 20th International Symposium Infoteh-Jahorina, Jahorina

[22] "Github project from Author's repository" (2021). [Online]. Available from: https://github.com/nikolicdanilo/JavaBackend-Static-Code-Analysis. Accessed: 20 July 2021 